Capítulo 8

\title{
Caracterización de las empresas del sector lácteo de Sincelejo, Sucre, Colombia, desde una perspectiva económica, social y tecnológica: un estudio de caso
}

\author{
Isaura Esther García Chávez ${ }^{1}$ \\ Piedad Martínez Carazo ${ }^{2}$ \\ María José Sierra Galindo ${ }^{3}$
}

\section{Introducción}

Colombia es un país con gran fortalecimiento en la industria de leche; a pesar de esto, ha venido presentando inconvenientes en el mercado, ya que los costos en los mercados a nivel internacional no están controlados. En cambio, a nivel nacional si están regulados, lo que deja en desventaja a los empresarios. Por otro lado, el comercio informal de productos derivados de la leche, ha impactado directamente el rendimiento del sector. Asimismo, se ha presentado un aumento en las importaciones de los productos derivados de la leche, lo que refleja la incompetencia de los productores nacionales para suministrar los insumos suficientes a todo el mercado interno. De esta manera, el sector se manifiesta pidiendo ayuda para fortalecer y desarrollar una mejor productividad. Y, en este sentido, el Ministerio de Comercio,

$1 \quad$ Asesora del Programa Ondas e investigadora adscrita al grupo Dimensiones Humanas de la Corporación Universitaria del Caribe- CECAR, Aspirante a Magister en Gestión de la Innovación, Psicóloga con experiencia en investigación

2 Doctora en Creación, Estrategia y Gestión de Empresas por la Universidad Autónoma de Barcelona (España); Magister en Creación, Estrategia y Gestión de Empresas; Especialista en Finanzas, Universidad de Cartagena; Especialista en Gerencia de la Hacienda Pública, Corporación Universitaria del Caribe; Administradora de Empresas, Universidad del Sinú. Asesora y miembros de la Sala de Fundadores de la Corporación Universitaria del Caribe - CECAR.

3 Magister en Gestión de la Innovación por la Universidad Tecnológica de Bolívar (Colombia); Diseñadora Industrial por la Universidad de Pamplona (Colombia). Docente catedrático de la Corporación Universitaria del Caribe - CECAR. Asistente de Laboratorio de Creatividad e Innovación de la Corporación Universitaria del Caribe - CECAR 
Industria y Turismo intervino a través del Programa de Transformación Productiva con resultados evidentes.

Por otro lado, la industria del Sector Lácteo en Colombia presenta un déficit hace unos años, y los comercializadores de leche con otros expertos manifiestan que el sector se encuentra en quiebra, lo que han conducido a una crisis por la falta de abastecimiento interno y que las industrias internacionales han cobrado más fuerza en el país. Los precios que les son pagados a los comercializadores de leche no aumentan desde hace más de 11 años; en cambio, los insumos para la producción aumentan descontroladamente cada año en el país. Sumado a esto, el Gobierno ha tenido poca inversión en Ciencia, Tecnología e Innovación en el sector, dejando al país rezagado en comparación con competidores externos. Por último, los convenios de libre comercio, que se firmaron hace un par de años, han llevado al sector lechero a una "crisis" que preocupa seriamente a sus comercializadores y productores (Robayo, 2018).

En el Departamento de Sucre, y en particular en la ciudad de Sincelejo, las empresas del Sector Lácteo presentan un bajo nivel de competitividad, tal como lo afirman Hernández, Bustamante y Porto (2014), quienes también destacan el alto grado de vulnerabilidad del sector; pues, cualquier cambio en el entorno, puede afectar al ganado, la leche y por ende la productividad. Además, destacan que es un subsector en el que existe un alto grado de rivalidad entre las empresas; pues están en constante búsqueda de estrategias como la Innovación para mejorar el producto y acaparar el mercado. Por lo tanto, los niveles de Innovación de las Empresas del Subsector Lácteo en la ciudad de Sincelejo y del Departamento deben ser intervenidos no solo para mejorar la competitividad local, sino para incursionar en mercados internacionales (Hernández et al., 2014).

Teniendo en cuenta la importancia que tiene la producción de leche y sus derivados en la economía de la región sucreña, es muy importante desarrollar estudios que mancomunadamente direccionen esfuerzos a la caracterización del Sector Lácteo desde los diferentes aspectos y contar con un panorama general de la situación económica, social y tecnológica de las empresas del sector con el fin de contribuir información relevante sobre el estado de las empresas y qué necesitan para mejorar los procesos internos que contribuyan al crecimiento en la competitividad y productividad del sector a nivel local, nacional e internacional. 
En este sentido, la caracterización de las empresas del sector en la ciudad de Sincelejo, desde la perspectiva económica, social y tecnológica, permitió identificar las falencias del sector en materia de inversión de Nuevas Tecnologías que soporten el crecimiento y estén a la vanguardia para la implementación de nuevos procesos industriales, que constituyan a la generación de nuevos procesos y productos de mejor calidad atractivos, tanto en mercados nacionales como internacionales. Para este fin, la Innovación se puede considerar como una herramienta fundamental en el mejoramiento de la competitividad del Sector Agroindustrial en la ciudad de Sincelejo, la cual se puede utilizar como una estrategia corporativa de las organizaciones empresariales y uno objetivos misionales del crecimiento de las empresas, dentro del marco de la globalización. En este sentido, la inversión en Tecnología, Innovación e Investigación, recobra importancia como herramienta capaz de impulsar hacia una competitividad de clase mundial (Rico, Martinez \& Rico, 2015).

\section{Antecedentes teóricos y conceptuales}

Muchos autores han tratado de definir e identificar el concepto de Innovación; si bien, no de forma explícita, sus argumentos se han convertido en el punto de partida para los conceptos y definiciones sobre Innovación desarrollados tiempos después. Por ejemplo, el autor Adam Smith, en su escrito literario Una investigación sobre la naturaleza y causas de la riqueza de las naciones, que fue publicado en 1776, muestra cómo a través de la cualificación, separación y especialización del trabajo se puede motivar la invención de nuevas herramientas y máquinas para el desarrollo de nuevos procesos y productos. En esta misma línea, el autor David Ricardo describe en su obra Principios de economía política y tributación, en el año (1817), cómo las remodelaciones técnicas y los nuevos descubrimientos, podrían contribuir a producir una cantidad de bienes, incluso a desarrollar más la producción empleando una menor cantidad de mano de obra (Formichella, 2005).

Por otro lado, Peter Drucker (1985), reconoce la Innovación como una herramienta particular que utilizan los Empresarios Emprendedores, por la cual se ven las transformaciones como oportunidades de nuevos negocios distintos a los tradicionales., "Es la acción de suministrar y utilizar 
los recursos como una nueva capacidad de producir riqueza. Ya que la Innovación crea recursos. En este sentido, no puede existir algo sin que el hombre le encuentra la aplicación de lo natural y entonces lo dota de valor económico". En este mismo orden de idea, manifiesta que el Empresario Innovador ve el cambio como algo saludable, que le traerá beneficios en un futuro, teniendo la capacidad de buscar el cambio, responder a él y lo explota como una oportunidad (Drucker, 1985).

Ahora bien, la Innovación va más allá de un concepto que se relaciona con algo nuevo; es algo más complejo que implica proponer, crear, producir y utilizar las estrategias de mercado ideales que van desde la renovación y transformación de lo actualmente comercializado, hasta lograr algo diferente y atractivo a los ojos de los clientes (Fernández \& Fernández, 1988). Es así, como se forma un engranaje entre los procesos, productos y servicios; organización, institucional y financiero de las grandes industrias.

En este sentido, los directivos de las empresas cumplen un rol fundamental para el establecimiento del éxito o el fracaso organizacional; como gestionadores tienen mayor potencia en el impulso de estrategias de organización que mejoren la eficiencia y la eficacia, para aprovechar las oportunidades de mercado, e identificar y neutralizar amenazas potenciales (Lepak \& Snell, 1999). Algunos estudios han descubierto que los altos grados de eficiencia y eficacia de la gestión aportan a las organizaciones una gran ventaja (Adner $\&$ Helfat, 2003), pero han dejado de lado que los directivos en los diferentes rangos jerárquicos tienen diferentes funciones que exigen múltiples habilidades para el Desarrollo Empresarial (Kraut, Pedigo, McKenna, \& Dunnette, 2005).

De acuerdo con lo anterior, reconocer la importancia que tiene el desarrollo de la Innovación en las Empresas, permite la generación de nuevos procesos que conducen a las organizaciones a mejorar su competitividad y hacerse visibles en el mercado. Asimismo, tener un diagnóstico sobre la situación de las empresas desde varias perspectivas, proporciona bases importantes que determinarán el rol que cumplirá la Innovación desde cada aspecto, tanto económico, social y tecnológico, para el aprovechamiento de los recursos y el fortalecimiento de las actividades generadas para el aumento de la productividad. 


\section{Metodología}

La metodología implementada es de carácter cualitativo, a través de la metodología de Estudio de Caso. Para determinar la población objeto de estudio de las tres empresas analizadas, se establecieron los siguientes criterios: Empresas productivas y activas en el sector lácteo, registradas en la Cámara de Comercio de Sincelejo e Invima, localizadas en el municipio de Sincelejo. Y, finalmente, que permitieran el acceso a la información. En la Tabla 1 se observan las empresas seleccionadas.

Tabla 15

Empresas Procesadoras de Lácteos en la ciudad de Sincelejo que cumplen con los criterios

\begin{tabular}{|c|c|c|}
\hline $\begin{array}{c}\text { Nombre o Razón } \\
\text { Social }\end{array}$ & $\begin{array}{l}\text { Productos } \\
\text { Elaborados }\end{array}$ & $\begin{array}{l}\text { Registro en Cámara de } \\
\text { Comercio y el Invima }\end{array}$ \\
\hline $\begin{array}{l}\text { Alimentos de Mi Costa } \\
\text { S.A.S. }\end{array}$ & Yogurt & SI \\
\hline $\begin{array}{l}\text { Lácteos Sincelejo Hnos. } \\
\text { S.A.S. }\end{array}$ & $\begin{array}{l}\text { Queso doble crema, } \\
\text { suero costeño }\end{array}$ & SI \\
\hline Empresa Prolasuc & $\begin{array}{l}\text { Leche entera, suero, } \\
\text { queso, crema de leche } \\
\text { y leche pasterizada }\end{array}$ & SI \\
\hline
\end{tabular}

Fuente: Elaboración propia a partir de la Cámara de Comercio de Sincelejo

El proceso de caracterización llevado a cabo en las tres Empresas del sector, estuvo dividido en dos fases: en la primera fase, se obtuvieron los datos generales de la empresa (creación, edad, propiedad del capital); seguido se identificaron los elementos corporativos (misión, visión, historia, estructura organizacional). Y en la segunda fase, se establecieron aspectos desde la perspectiva económica (en la cual se tuvo en cuenta: financiera, producción, marketing, gestión de calidad), la perspectiva social y la perspectiva tecnológica. Para poder obtener la información y organizarla desde las diferentes perspectivas, se utilizó como herramienta de recolección de información, la aplicación de entrevista semiestructurada, la observación y revisión literaria. 
Caracterización de las empresas del sector lácteo de Sincelejo, Sucre, Colombia, desde una perspectiva económica, social y tecnológica: un estudio de caso

\section{Resultados}

A continuación, se evidencian, mediante las Tablas 1,2 y 3, la caracterización de las tres empresas, desde la perspectiva económica, social y tecnológica.

Tabla 16

Empresa Alimentos de Mi Costa S.A.S.

Datos Generales

de la Empresa

Creación: 2013. Edad: 6 años. Propiedad del Capital:

Privada. Tamaño: Pequeña

Misión: Somos una organización que se dedica a la comercialización y producción de productos derivados de la leche como el yogurt entero con dulce con fruta natural, reconocida a nivel Caribe por la calidad del producto y servicio que se ofrece, brindándole a la Región una alternativa alimenticia con excelentes contenidos naturales y nutricionales.

Visión: Para el 2020, ser una de las mejores empresas productoras de yogurt entero, con dulce de fruta natural de la Región Caribe, organizada y tecnificada, a fin de brindar la más excelente oportunidad alimenticia con altos estándares de calidad.

Historia: La empresa fue creada por la idea de unos paisas que llegaron a la ciudad con una receta sobre como elaborar

Elementos yogur; inicialmente, el proceso era artesanal, donde se aplicaba Corporativos el conocimiento de manera empírica. Con el tiempo, al ver que el producto iba teniendo aceptación en el mercado la idea fue mejorando por parte de sus fundadores. En marzo de 2016, se constituye legalmente la empresa con el nombre Distrilácteos La Carolina S.A.S. ante la Cámara de Comercio de Sincelejo, pero, después de 3 años, la empresa modifica su nombre a Alimentos de Mi Costa S.A.S., quedando con la misma razón social y se amplía la capacidad instalada con la adquisición de nuevas maquinarias que han permitido la obtención de un producto de mejor calidad.

Estructura Organizacional: La empresa, conformada por 3 Áreas y 7 Empleados (5 hombres y 1 una mujer) distribuidos de la siguiente manera:

Área Administrativa: (1 profesional y una técnica) 2. Área Operativa (4 técnicos). 3. Ventas (1 bachiller) 


\title{
Datos Generales
}

\author{
de la Empresa
}

\section{Creación: 2013. Edad: 6 años. Propiedad del Capital: Privada. Tamaño: Pequeña}

Financiera: La empresa inicia con poco capital, el cual se fue incrementando con el transcurso de los últimos años, producto del apalancamiento financiero a través del sector bancario y acreedores, hasta alcanzar una estabilidad financiera, un capital de trabajo adecuado, un patrimonio importante, y un crecimiento de las ventas que le ha permitido obtener un posicionamiento en el mercado.

Producción: Inicialmente funcionaba con una máquina de pedal que era la que sellaba las bolsas de yogurt. Producto del crecimiento y desarrollo de la organización, ha mejorado la calidad de sus productos y ha adquirido nueva maquinaria con tecnología avanzada, denominada Signa (envasadora que dosifican).

Cada producto tiene su propia imagen y presentación (etiquetas con la información nutricional y contenido del producto, empaque), que se elabora a partir de una ficha técnica que contiene la información sobre el diseño del producto, para alcanzar estándares del mismo.

\section{Perspectiva Económica}

Marketing: Los canales de distribución son: ventas directas al consumidor final, mayoristas, minoristas, agentes comerciales, almacenes de cadena. Los precios son fijados a través de la negociación con los distribuidores. Durante los últimos tres años la Empresa ha incrementado las ventas de manera importante al pasar de vender mensualmente, 6.000 a 7.000 litros de yogurt. Actualmente las ventas han ascendido a 15.000 litros, aproximadamente, como resultado de la calidad de los productos. Es decir, que durante los últimos dos años las ventas se han incrementado en un $100 \%$.

La estrategia de promoción y publicidad utilizada consiste en anuncios en la televisión local, carteleras, folletos, entre otros. Actualmente, el principal mercado es el Departamento de Sucre. Sin embargo, la empresa está penetrando al Departamento de Córdoba y se está proyectando hacia otros Departamentos del territorio nacional. No implementa la estrategia de segmentación de mercados.

\section{Gestión de la Calidad}

La empresa cuenta con un sistema de control de calidad, donde tienen procedimiento para garantizar la higiene, manejo de residuos y calidad de los proveedores, para garantizar un producto con todas las normas de salubridad. 


\section{Datos Generales}

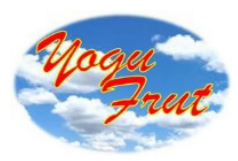

de la Empresa

Creación: 2013. Edad: 6 años. Propiedad del Capital:

Privada. Tamaño: Pequeña

La empresa contribuye a la generación de nuevo conocimiento, a través del apoyo a la investigación científica y aplicada que realizan las Instituciones de Educación Superior del municipio de Sincelejo.

La empresa genera un impacto social positivo en el Departamento de Sucre, contribuyendo a la generación de empleo, vinculando, a su cadena de suministro, a vendedores

Perspectiva Social Independientes que lo distribuyen otras localidades del Departamento de Sucre.

Produce alimentos saludables, que son supervisados por la Superintendencia de Industria Comercio y el Invima. Proyecta generar más empleos que permitan beneficiar a más familias, a través de una ampliación de cobertura, diversificación del portafolio de productos y servicios, y ampliación de los canales de distribución a nivel local y nacional.

La empresa apoya sus procesos desde dispositivos que comunican toda la gestión interna de la Empresa, mediante la

Perspectiva utilización de celulares móviles.

Tecnológica En el año 2016, para ajustarse a los parámetros exigidos por el Invima, como ente de control de los procesos productivos, implementó la estrategia de crear dos grandes albercas para la mejora de la producción y calidad de los productos.

Fuente: Elaboración propia a partir de la información suministrado por la empresa en la entrevista 
Tabla 17

Empresa Lácteos Sincelejo Hnos. S.A.S.

Datos Generales

de la Empresa

Creación: 2000. Edad: 20 años. Propiedad del Capital: Privada. Tamaño: Pequeña

Misión: Somos una empresa comercializadora y productora de productos derivados de la leche. Para ello, trabajamos con calidad, soportados con la tecnología apropiada y excelente talento humano de experiencia, comprometidos con los valores, corporativos y la preservación del medio ambiente.

Visión: Lácteos Sincelejo se proyecta para el año 2030 como la mejor empresa en el Departamento de Sucre, productora de productos lácteos y que proyecta incursionar en los mercados nacionales e internacionales.

Elementos Corporativos

Perspectiva Económica
Historia: Inicia de la idea de un empresario apasionado por la diversidad de productos que se pueden derivar de la leche, tuvo la idea de aprovechar la leche que él mismo transportaba, como una fuente de ingreso con valor agregado. Producto de ese trabajo se crea Lácteos Sincelejo como persona natural; ha sido una constante en sus procesos productivos. Sin embargo, en el año 2017, el señor Roger Barrera decide convertir su empresa de persona natural a persona jurídica, convirtiéndose en Lácteos Sincelejo Hnos. S.A.S., con el fin de organizar más la empresa en materia tributaria y legal.

Estructura Organizacional: La empresa está conformada por 21 empleados, distribuidos en las 4 áreas productivas.

Financiera: La empresa inicia con poca participación en el mercado y bajo volumen de ventas. Sin embargo, gracias a la visión de sus propietarios, la empresa se consolidó en el sector, logrando estabilidad económica, crecimiento de las ventas y ampliación del mercado. Actualmente, la empresa cuenta con capital propio suficiente que les permite realizar inversiones sin recurrir a la financiación externa. 


\section{Datos Generales}

\section{de la Empresa}

Creación: 2000. Edad: 20 años. Propiedad del Capital: Privada. Tamaño: Pequeña

Producción: La empresa se abastece de varios canales de distribución en los municipios de la región sucreña y cordobesa; entre esos tiene a Sincé, San Pedro, Betulia, Chinú, Galeras, donde ganaderos con sus buenas prácticas suministran leche apta para procesar exclusivamente a esta. Anualmente, reciben un promedio de 20.000 litros de leche, suministrados por los ganaderos de la región, a través de un contrato con acuerdos de pago quincenal.

Los productos van dirigidos al mercado nacional y local. El 10\% de sus ventas se realiza directamente al consumidor final en el punto de la empresa y el $90 \%$ restante se distribuye a través de

Perspectiva Económica mayoristas. Los precios son negociables con sus clientes, dentro de los cuales se destacan Crepes and Wafles, supermercados y hoteles.

Marketing: La estrategia de publicidad utilizada es la presentación de portafolios a diferentes locales comerciales que distribuyen y venden productos lácteos. También, se apoyan en la publicidad VOz a voz, que consiste en ofrecer al cliente un producto de calidad y ese mismo cliente los recomiende, siendo esta una de las estrategias de marketing más importantes. La empresa se proyecta ampliar sus canales de distribución a nivel local, nacional e internacional, a través de un proyecto de internacionalización.

Gestión de la Calidad: La empresa cuenta con un sistema de control de calidad, donde tienen procedimiento para garantizar la higiene, manejo de residuos y calidad de los proveedores, para garantizar un producto con todas las normas de salubridad. 


\section{Datos Generales}

\section{de la Empresa}

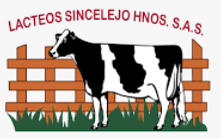

Creación: 2000. Edad: 20 años. Propiedad del Capital: Privada.

Tamaño: Pequeña

La Empresa ejerce la responsabilidad social, a través de la cual logra impactar en la sociedad con la generación de empleo que beneficia a varias familias del municipio de Sincelejo. Apoya obras

Perspectiva Social sociales de la comunidad como, por ejemplo, entrega de bonos, rifas y regalos, que son utilizadas en la realización de actividades que buscan un bien común entre la comunidad donde se encuentra ubicada la empresa.

La empresa apoya sus procesos en los programas de Microsoft Office diseñados a la medida o CNC (Control Numérico Computarizado). Renovaron dos de sus cuartos fríos, optimizando su sistema eléctrico. Cuentan con un sistema de facturación electrónico especializado que facilita la contabilidad de la empresa.

\section{Perspectiva Tecnológica} Asimismo, están en procura de implementar nuevos softwares que permitan que toda la información manejada en la empresa sobre proveedores, clientes y procesos internos estén anclados a un sistema que ayude, de manera sistemática, a organizar toda la información y evitar el manejo de documentos en físico. Lo anterior ayudará a recuperar espacios dentro de la empresa que son ocupados por cantidades de archivos en físico.

Cuenta con una planta especializada en el área de producción que permite tener el control de todo el procesamiento de la leche y la elaboración de productos. Poseen un laboratorio interno que permite evaluar los niveles de la calidad de la leche que reciben de los proveedores y, cuando necesitan un estudio más especializado, se apoyan en laboratorios externos, que garantizan la efectividad en la calidad de la materia prima.

Fuente: Elaboración propia a partir de la información suministrado por la empresa en la entrevista 


\section{Tabla 19}

Empresa Prolasuc

Datos generales

de la Empresa
PRODUCTOS LACTEOS DE SUCRE PROLASUC

Lider en Calidad y Sabor

Creación: 1986. Edad: 33 años. Propiedad del capital: Privada. Tamaño: Pequeña

Misión: Somos una empresa familiar, líder en la producción y comercialización de leche y derivados lácteos, con cobertura a nivel nacional y un alto reconocimiento a nivel regional, por su excelencia en los diferentes productos, apoyados en un equipo humano calificado, con un alto compromiso con la satisfacción del cliente y aliados estratégicos, aplicando procesos tecnológicos adecuados, siendo social y ambientalmente responsables.

Visión: En el año 2023 seremos reconocidos como la Empresa líder en la producción y comercialización de leche y sus derivados en la Región Caribe colombiana y tendremos un significativo reconocimiento en el mercado nacional.

Historia: Creada en la ciudad de Sincelejo, en el año 1986, con miras al aprovechamiento de la producción lechera del Departamento de Sucre, por el gerente, quien siendo profesor de

Elementos Corporativos un colegio de la ciudad comenzó a realizar pruebas de queso en el laboratorio con los estudiantes de la institución; después de varios intentos y experimentación se dio cuenta de que el producto que daba como resultado de esos experimentos le salía bien. En ese momento, decidió comercializar el producto desde su casa con un capital pequeño y distribuía el producto a amigos y familiares que fueron dándole publicidad por la calidad y eso permitió ser reconocido en el mercado, expandiendo sus clientes, ofreciendo solo queso costeño. Los ingresos que obtenía de su labor como profesor los fue invirtiendo con ayuda de su esposa, quien también trabajaba y aportaba; los ingresos no eran suficientes y tuvieron que acceder a préstamos bancarios para fortalecer el capital y lograr comprar un lote donde construyeron una bodega que, con el tiempo, por el aumento de sus clientes a nivel local, regional y nacional, se fue posesionando como una de las empresas del sector con mayor producción. 


\section{Datos generales}

de la Empresa

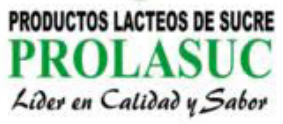

Creación: 1986. Edad: 33 años. Propiedad del capital: Privada. Tamaño: Pequeña

Actualmente, tiene sede en Bogotá y Barranquilla, maneja diferentes gamas de productos y cuenta con una capacidad instalada para proveer a diferentes centros comerciales como el Éxito.

\section{Elementos Corporativos}

Estructura Organizacional: Conformada por 3 áreas: 1. Administrativa (7 profesionales y 4 técnicos), 2. Operativa (6 profesionales, 10 técnicos y 10 bachilleres) y 3 . Ventas 2 profesionales y 2 técnicos)

Financiera: Es una empresa que empezó con poco capital y tuvo que acceder a préstamos bancarios para poder cumplir los requerimientos del mercado. Actualmente, cuenta con su propio capital, producto del incremento de sus ventas, lo que ha permitido cumplir con las responsabilidades adquiridas y lograr una estabilidad financiera para sus inversiones y nuevos proyectos.

Producción: La capacidad de producción sobrepasa los 40.000 litros diarios de leche y con todo tipo de tecnologías que mantiene la inocuidad del producto. Los productos van dirigidos hacia el mercado nacional y se utiliza estrategia de segmentación; en tanto,

Perspectiva Económica existe una línea de productos estándar, y una premium destinada a clientes más sofisticados, como hoteles. El 10\% de sus ventas se realiza directamente en el punto de fábrica, el $55 \%$ a mayoristas y el 35\% restante a intermediarios, que distribuyen directamente al consumidos o a tiendas minoristas.

Marketing: La estrategia de publicidad utilizada son la presentación de portafolios a diferentes locales comerciales que distribuyen y venden productos lácteos. Asimismo, utilizan como publicidad los canales locales de televisión.

Gestión de la Calidad: La empresa cuenta con un sistema de control de calidad, donde tienen procedimiento para garantizar la higiene, manejo de residuos y calidad de los proveedores, para garantizar un producto con todas las normas de salubridad. 
Datos generales

de la Empresa
PRODUCTOS LACTEOS DE SUCRE

PROLASUC

Lider en Calidad y Sabor

Creación: 1986. Edad: 33 años. Propiedad del capital: Privada. Tamaño: Pequeña

\section{Perspectiva Social}

Perspectiva

Tecnológica
Generación de empleo y apoyo a las gestiones sociales de la comunidad.

Fuente: Elaboración propia a partir de la información suministrado por la Empresa en la entrevista

\section{Conclusiones}

Desde la perspectiva económica, son empresas que en sus inicios no contaban con el capital necesario para su organización, y tuvieron que recurrir a préstamos bancarios para lograr ir creciendo y atender las demandas del mercado. Actualmente, han aumentado su producción y ventas logrando un aumento de sus ingresos. Sin embargo, presentan deficiencias en la obtención de nuevos productos, por la poca capacidad instalada con la que cuentan. Utilizan como estrategias de marketing la presentación de portafolio a sus clientes, publicidad televisiva local y preventistas, tienda a tienda, ofreciendo los productos, siendo reconocidas en la ciudad.

Desde la perspectiva social, las empresas brindan aportes a actividades sociales en favor del beneficio de la comunidad; permiten el desarrollo de investigaciones científicas de carácter académico, que contribuyen en la obtención de nuevos conocimientos y generación de nuevas ideas.

Desde la perspectiva tecnológica, cuentan con plantas especializadas de poca capacidad tecnológica que se encargan de procesar la materia prima convirtiéndola en un producto de calidad con los controles de salubridad y de higiene. 
Finalmente, las tres empresas del sector requieren mejorar sus procesos internos, invertir en Nuevas Tecnologías, que permitan el crecimiento en la implementación de procesos, productos y servicios, para mejorar la competitividad. Asimismo, las empresas requieren que su personal mantenga capacitándose para que estén a la vanguardia de lo que demanda el mercado nacional y poder proyectarse para penetrar a mercados internacionales.

\section{Referencias}

Adner, R., \& Helfat, C. E. (2003). Corporate effects and dynamic managerial capabilities. Strategic Management Journal, 24(10), 1011-1025. https://doi.org/10.1002/smj.331

Cámara de Comercio de Bogotá. (2018, Julio). ¿Cómo va el sector Lácteo? Cluster Lácteo de Bogotá-Región. https://www.ccb.org.co/Clusters/ Cluster-Lacteo-de-Bogota-Region/Noticias/2018/Julio-2018/Comova-el-sector-Lacteo

Cámara de Comercio de Sincelejo. (2018). Informe Económico del Departamento de Sucre. Sincelejo.

Drucker, P. (1985). La Innovación y el Empresario innovador. Edhasa.

Formichella, M. (2005). La evolución del concepto de Innovación y su relación con el desarrollo. Instituto Nacional de Tecnología Agropecuaria. https://www.t-educa.cl/descargas/pdfs_portal/emprendimiento/ emprendydesarrollolocal.pdf

Fernández, E., \& Fernández, Z. (1988). Manual de dirección estratégica de la tecnología. Ariel Economía.

Hernandez, F., Bustamante, L., \& Porto, I. (2014). Evaluación del grado de competitividad de cinco Empresas del sector lechero de Sincelejo Colombia. Investigación, Desarrollo e Innovación, 5(1), 8-19. https://doi.org/10.19053/20278306.3135

Kraut, A. I., Pedigo, P. R., McKenna, D. D., \& Dunnette, M. D. (2005). The role of the manager: What's really important in different management jobs. Academy of Management Executive, 19(4), 122-129. https://doi.org/10.5465/AME.2005.19417914

Lepak, D. P., \& Snell, S. A. (1999). The human resource architecture: Toward a theory of human capital allocation and development. Acad- 
emy of management review, 24(1), 31-48. https://doi.org/10.5465/ amr.1999.1580439

Robayo, M. (2018). Creación de una Empresa para la producción; recolección y comercialización de leche bovina en el municipio de Turmequé [Tesis de pregrado, Universidad Católica de Colombia] Repositorio Ucatólica. https://repository.ucatolica.edu.co/bitstream/10983/16091/1/ CREACI\%C3\%93N\%20DE\%20UNA\%20EMPRESA\%20PARA\%20 LA\%20PRODUCCI\%C3\%93N_\%20RECOLECCI\%C3\%93N\%20 Y\%20COMERCIALIZACI\%C3\%93N\%20DE\%20LECHE\%20BOVINA\%20EN\%20EL\%20.pdf

Rico, H; Martinez, Z; \& Rico, F. (2015). Comportamiento innovador del sector del sector alimentos en el distrito de barranquilla. Universidad Simon Bolivar. https://bonga.unisimon.edu.co/handle/20.500.12442/1193 